\title{
Developing a Gait Enhancing Mobile Shoe to Alter Over-Ground Walking Coordination
}

\author{
Ismet Handzic, Erin Vasudevan, and Kyle B. Reed
}

\begin{abstract}
This paper presents a Gait Enhancing Mobile Shoe (GEMS) that mimics the desirable kinematics of a splitbelt treadmill except that it does so over ground. Split-belt treadmills, with two separate treads running at different speeds, have been found useful in the rehabilitation of persons with asymmetric walking patterns. Although in preliminary testing, beneficial after-effects have been recorded, various drawbacks include the stationary nature of the split-belt treadmill and the inability to keep a person on the split-belt treadmill for an extended period of time. For this reason, the after-effects for long-term gait training are still unknown. The mobile ability of the GEMS outlined in this paper enables it to be worn in different environments such as in one's own house and also enables it to be worn for a longer period of time since the GEMS is completely passive. Healthy subject testing has demonstrated that wearing this shoe for twenty minutes can alter the wearer's gait and will generate after-effects in a similar manner as a split-belt treadmill does.
\end{abstract}

\section{INTRODUCTION}

Asymmetric gait is sometimes developed in individuals with central nervous system damage, such as stroke, or persons who have suffered damage to the spinal cord, brainstem, cerebellum, or motor cortex. In such cases, a limp is developed where the person does not fully extend their foot backward far enough, which can prevent them from effectively pushing off into the swing phase.

Previous split-belt treadmill research has demonstrated both altered human walking patterns [1-5] and animal gait [6][7] with even and uneven walking patterns. This adaptation process occurs when the split-belt's two treads are run at different speeds so that the faster tread is repeatedly moving one leg backward faster than the other during the stance phases. Once the treads are returned to the same speed, an altered walking pattern is retained, which is called an after-effect. This continuous and repeated splitbelt gait training has been found to temporarily restore a normal walking pattern [8]. However, individuals with such corrected walking patterns can only retain it for a short period of time and the gait pattern does not effectively transfer to walking over ground. Because adaptation effects only lasted for a short period of time in these studies, the effects of long-term training are still unknown.

The concept of a mobile shoe that can mimic the kinematics of a split-belt treadmill has been developed into the Gait Enhancing Mobile Shoe (GEMS), shown in Figure 1. While walking over ground, the GEMS

I. Handzic and K. Reed are in the Department of Mechanical Engineering at the University of South Florida.

E-mail: ihandzic@mail.usf.edu \& kylereed@usf.edu

E. Vasudevan is with Moss Rehabilitation Research Institute.

E-mail: VasudevE@einstein.edu successfully mimics the relative foot motion an individual on a stationary split-belt treadmill undergoes.

The GEM Shoe presented in this paper is the successor of previous versions [12][19]. The first GEMS [12] was passive, but had no control of the backward foot motion. Although it moved the wearer's foot backward, it did so in a jerky and fairly unpredictable motion comparable to sliding on ice or a slippery surface. The second GEMS [19] provided a smooth motion by controlling the generated horizontal motion. However, because of the various motion controls, this version ended up being too high off the ground and too heavy for actual subject testing. The version described here regulates the shoe travel velocity using unidirectional dampers. This shoe is completely passive, thus no power source is required and it can be worn in different environments including one's own home and for extended periods of time. This possibility of wearing the GEMS for extended periods of time increases the chance to produce positive gait correcting after-affects.

\section{BACKGROUND}

\section{A. Gait Phases and Reaction Forces}

Since this paper presents a gait correction method based on the Gait Enhancing Mobile Shoe (GEMS), it is important to review the basics of a normal human gait pattern. As shown in Figure 2, the gait cycle can be divided into two distinct phases: the stance phase and the swing phase [14]. The analysis of the shoe is predominantly concerned with the motion and forces involved during stance phase.

The graph in Figure 3 illustrates the horizontal and vertical forces applied from the foot during a typical adult gait starting at heel contact. During the stance phase, a

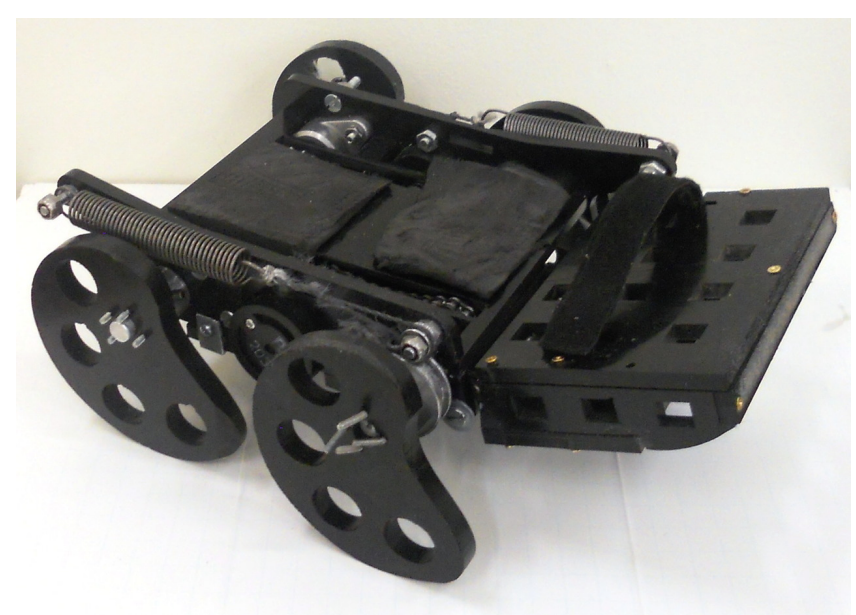

Fig. 1. Gait Enhancing Mobile Shoe designed to move one foot backward during the stance phase for use in correcting asymmetric gaits. 


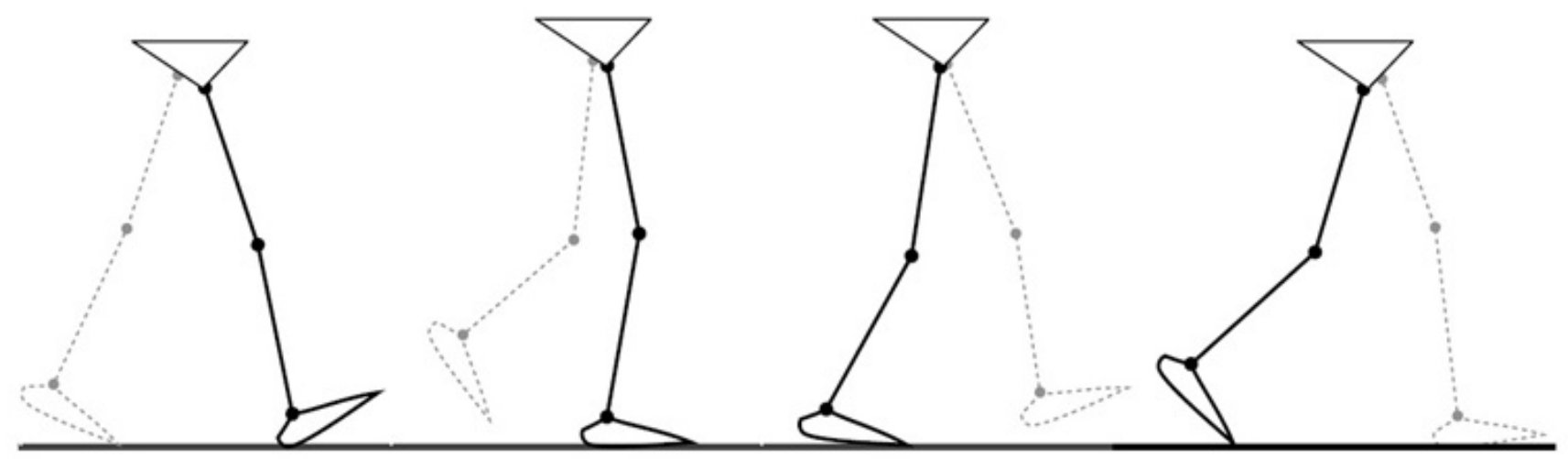

Fig. 2. Adult Human Walking Gait Cycle. Dark lines show the stance phase, and the dotted lines show toe off, swing phase, and heel contact.

slightly variable vertical force is applied while, at $33 \%$ of the gait cycle, the horizontal reaction force switches from accelerating the body backward to accelerating the body forward. To generate the necessary horizontal forces, the GEMS passively converts the vertical force from the wearer during the stance phase and redirects it into a horizontal motion. At heel contact the wheels of the GEMS starts pushing the wearer's foot backward similar to stepping on a moving treadmill. While in the stance phase, the GEMS redirects the downward force into a controlled horizontal backward motion. The avoidance of the body's balancing reflexes is a critical aspect of the GEMS, so smooth motion and consistency are ideal.

\section{B. Correcting Gait}

Training on a split-belt treadmill has been shown to successfully alter the walking pattern of individuals with an asymmetric gait arising from a stroke or damage to the central nervous system [1-5]. An asymmetric gait occurs when the step length of one leg is longer than the step length of the other leg. In hemiplegic test subjects, the gait

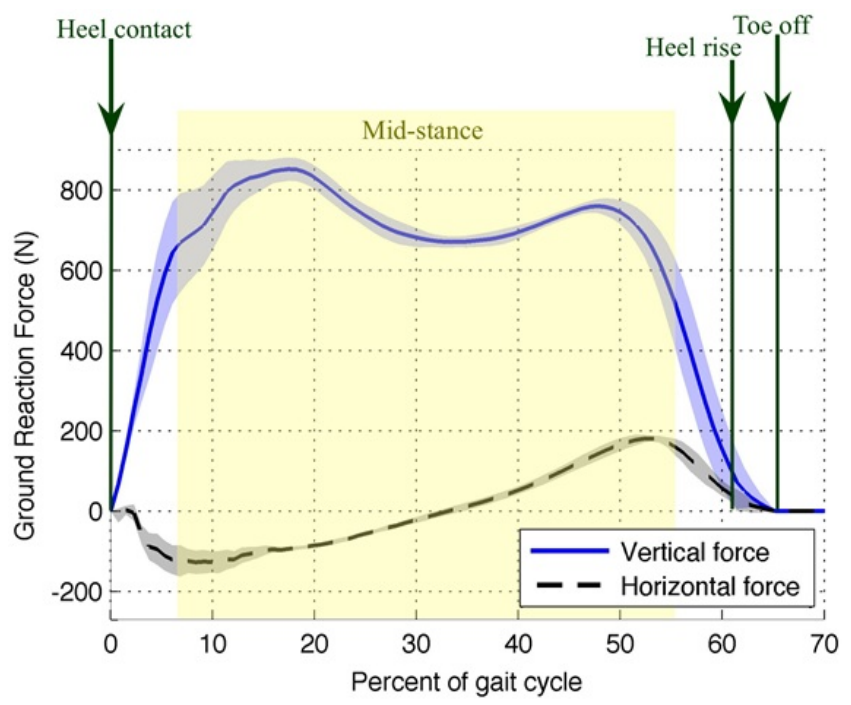

Fig. 3. The GEMS uses the changing vertical and horizontal forces throughout the gait cycle to generate the desired motion passively. is corrected by attaching the GEMS to the leg that has a shorter step. This exaggerated motion prompts the subject to take longer strides with the hemiplegic leg. The limitation of this beneficial after-effect is that it diminishes quickly during over-ground walking. Due to the time-limited nature of the split-belt treadmill, long-term after-effects need to be investigated by long-term adaptation studies. This long-term investigation can easily be conducted by having a mobile device such as the GEMS by allowing individuals to wear the shoe over a longer time period, periodically observing after-effects.

Since this research is based on previous split-belt treadmill gait studies, it is important to point out how walking on the GEMS differs from walking on a split-belt treadmill with asymmetric belt velocities. While the body's velocity relative to ground is zero on a split belt treadmill, the relative velocity of the GEMS is non-zero and forward. The GEMS forces the wearer's foot backward whereas the stationary foot has a zero velocity relative to ground. For both the split belt treadmill and the GEMS, the relative velocity between both feet is similar and the GEMS takes the place of the faster tread. To assess the differences between these environments, future research includes using an asymmetric passive dynamic walker [15] to study the differences between walking on the GEMS, a split belt treadmill, and walking over ground.

Based on our theory, prolonged use of the GEMS should yield positive after-effects in individuals with asymmetric gait and allow individuals to develop a more persistent symmetric gait. Training an individual with the GEMS may also strengthen muscles due to the different walking pattern that is developed [16], which in turn could alter the individual's gait.

The benefits of the GEMS are not only in building longterm after-effects but the shoe can also be used to compensate for the asymmetric walking pattern by fitting the shoe onto the healthy leg. By doing this, the healthy leg is pushed further back, which evens out the step length of both sides, mimicking a normal gait. Compensation may work, but is a less ideal solution since it requires the individual to always wear the shoe. Generating an after-effect is the ultimate goal as this will restore motion in the individual. 


\section{GEMS and Context Awareness}

The concept of context awareness [9] is important in our discussion of the GEMS. Context awareness is the body's ability to unconsciously anticipate the environment around them while preparing for disturbances [17]. This can easily be witnessed in situations where the body relies on visual cues [18], such as stepping onto an escalator [10][11]. In particular, as an individual approaches a stationary (i.e., broken) escalator, the body will automatically adjust to engage the escalator as they typically do by leaning forward, but, since the escalator is not moving, the person becomes unbalanced. The body's internal model expects to lean forward when getting on the escalator because of the many previous interactions with it, but the person stumbles because there is no acceleration. Context awareness shows us evidence of visual exteroception and its role it plays in the conditioning of the human gait and gait adaptation process in symmetric gait in hemiplegic patients.

It is this context awareness that is hypothesized to be an integrating factor in the inability to store the previously described feed forward motion learned in splitbelt gait manipulation research [1]. While after-effects can be achieved [1][12], as subjects adapt to the asymmetric treadmill speed and subsequently walk over ground, the learned gait motion disappears quickly. The visual cues are distinctly different between walking on a treadmill and walking over ground.

The concept of the GEMS eliminates the problem of context awareness during the gait adaptation process. With the GEMS, the human gait is altered in the natural context of walking. The walking gait is slowly altered using the splitbelt treadmill concept, however during conditioning with the shoe, there is no disconnect between the visual cues during the conditioning process and the visual cues after the adaptation process.

\section{GEMS DESIGN}

\section{A. First GEMS Design}

The first version of the Gait Enhancing Mobile Shoe (GEMS) [12] was simple and successful at pushing the wearer's foot backward relative to the opposite foot. However, due to this version's lack of control and damping, the backward motion was very jerky and variable, unlike a split-belt treadmill and more like slipping on ice. Although this version evoked some short term after-effects, it is hypothesized that the uncontrolled and variable motion of this version triggers the body's balancing, which in turn prohibits any long term after-effects. While it was a simple and durable design, this version also had low adjustability in backward motion, velocity, and travel distance.

\section{B. Second GEMS Design}

The second version [19] also generated the backward motion passively, but by using a magnetic particle brake and a microcontroller circuit. The brake controlled the damping in the shoe to mimic changes in force and direction typically observed during the respective gait cycle phase. Instead of using traditional wheels, it utilized Archimedean spiral shaped wheels, which convert the downward forces from the wearer into a horizontal motion. Although the second version produced a smooth transition and a constant shoe velocity comparable to a split-belt treadmill, it was too heavy, too tall, and somewhat unreliable for extensive human testing.

\section{Current GEMS Design}

The first GEMS was over simplified, lacked adjustability, and moved in a jerky unpredictable manner without any damping. The second GEMS was complicated, heavy, and too far off the ground. The third GEMS splits the design difference with a design that's completely passive, low to the ground, light, and provides sufficient damping for a constant velocity shoe travel. This version is also reliable enough to enable twenty minutes of training to test adaptation effects.

\section{GEMS Wheel Shape}

The wheel shape is an essential aspect of the GEMS design. The wheel shape is based on the concept of the Archimedean spiral shown in Figure 4, which is similar to the wheel used in [19]. When attached to an axle, this type of shape redirects the wearer's downward force $\left(F_{V}\right)$ during the stance phase into a horizontal backward motion. This resulting motion is similar to a circular wheel rolling down a hill, only that the slope is attached to the foot. The shape of the wheel and the generated horizontal force $\left(F_{H}\right)$ are determined by (1) and (2), respectively:

$$
\begin{gathered}
R(\theta)=b \theta^{\frac{1}{n}} \\
F_{H, a v g}=\frac{1}{R_{2}-R_{1}} \int_{R_{1}}^{R_{2}} F_{H}(R) d r
\end{gathered}
$$

where

$$
F_{H}=F_{V} *\left(\frac{L}{R}\right) .
$$

Parameters of the GEMS wheels are shown in Figure 4. Changing the slope at different points will allow for the optimization of the force to be generated during each instant during the stance phase assuming a linear increase in the size of the wheel $(n=1)$ since the relationship is based on the slope of the wheel in polar coordinates. This is not considered in this paper, but will be done in future work.

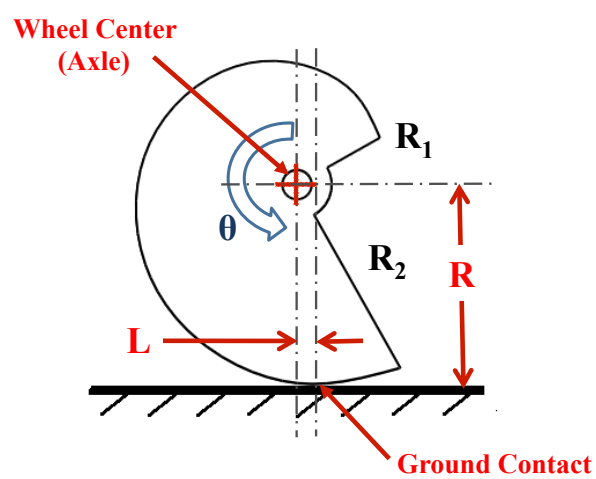

Fig. 4. GEMS Wheel shape. $R$ is the instantaneous radius and $L$ is the distance from ground contact to wheel center. 
The wheel parameters were chosen such that the largest radius was 2.75 in $(7.0 \mathrm{~cm})$, the shortest radius was 1.0 in $(2.54 \mathrm{~cm})$, and $n=1$ so that the wheels would generate an average horizontal backward force of $36 \mathrm{lb}(160 \mathrm{~N})$ given an $180 \mathrm{~N}(800 \mathrm{lb})$ vertical downward force from the wearer.

This wheel shape and shoe design allow the freedom to turn the wheels around so the shoe moves forward instead of backward. A setup with one foot moving forward and one foot moving backward during their respective stance phases would generate the greatest motion differential between both feet. This paper concentrates on the design of the shoe and the evaluation based on using one shoe pushing one leg backward. Multiple opposite direction shoes will be evaluated in a more comprehensive user study.

\section{E. Shoe Frame Structure}

The shoe structure is manufactured with delrin plastic cut using a laser cutter. Using delrin plastic for the frame allowed for a light and strong frame and a more rapid manufacturing process. The weight was reduced by extracting material from certain regions of the frame. The frame consists of a front half and a back half connected by a hinge, which is placed near the ball of the wearer's foot and is able to angle up to thirty degrees upward. Unlike previous GEMS, this version has a more natural feel to it by letting it deform with the shoe as the user toes off and the two hinged parts angle toward each other. When the user first initiates heel contact, the wearer's shoe bottom sits 3.4 in $(8.6 \mathrm{~cm})$ off the ground. Then as the wheels are bottomed out at the end of the stance phase, the wearer's shoe bottom sits 1.5 in $(3.8 \mathrm{~cm})$ off the ground. Also unlike the second GEMS design this version is significantly lighter at $3.3 \mathrm{lb}(1.5 \mathrm{~kg})$. The overall dimension of the GEMS are $11.0 \mathrm{in}(28.0 \mathrm{~cm})$ long, 7.9 in $(20.0 \mathrm{~cm})$ wide, and $2.0 \mathrm{in}(5.0 \mathrm{~cm})$.

\section{F. Unidirectional Dampers and Reset Mechanism}

A set of unidirectional dampers limit the backward speed and prevent a jerky motion once the user steps on the shoe. The unidirectional damper is basically an over-running clutch, which in turn can be described similar to a continuous ratchet mechanism; it allows rotation in one direction and is damped in the opposite direction. By finding the torque exerted by the wheels onto each axle, the dampers were sized to $17 \mathrm{lb}$-in $(1.9 \mathrm{~N}-\mathrm{m})$ per axle, which provides sufficient damping to decrease the velocity of the motion. The torque is sized to match a $180 \mathrm{lb}(82 \mathrm{~kg})$ person, but works for a range of $150 \mathrm{lb}(68 \mathrm{~kg})$ to $190 \mathrm{lb}(86 \mathrm{~kg})$ and can be adjusted as needed for other wearers. In order to keep the GEMS velocity steady for users out of this weight range, the damper must be replaced by a weaker damper for lighter wearers or a stronger one for heavier wearers. The generated horizontal force scales with the wearer's weight; the damper force is independent of the weight.

The dampers are coupled to the axles of the GEMS with a chain and two sprockets, one on each axle and one on each damper. As the wearer steps onto the shoe, the wheels apply a torque onto both axels which in turn are coupled to the

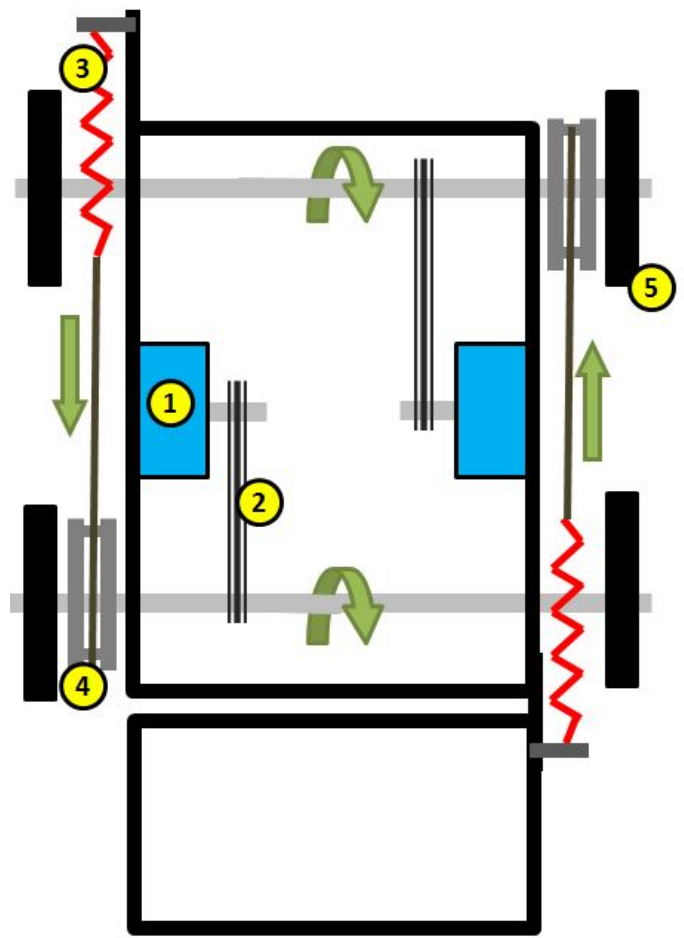

Fig. 5. GEMS Top view schematic of Unidirectional Dampers and Reset Mechanism. 1. Unidirectional Damper, 2. Steel Chain, 3. Reset Spring, 4. Reset Pulley, 5. GEMS Wheel

dampers. Once stance phase is initiated, the spring resets the shoe to its original base position ready for heel contact. The dampers used are unidirectional with an over-running clutch that exerts a damping force to one direction of wheel rotation but do not damp the mechanism when the shoe is resetting back to its initial state in preparation for another step. The setup of the unidirectional damper and the reset mechanism can be seen in Figure 5.

Once the GEMS completes the backward motion and the wearer toes off, the whole mechanism is reset. This is done by a set of springs that are attached to pulleys that in turn are attached to the axles themselves. This allows for the shoe dynamics to be identical every step.

\section{G. Adjustable Walking Platform}

In order to prevent any additional asymmetries not associated with the movement of the shoe, a walking platform was constructed to match the height and weight of the GEMS when only one GEMS was worn. This platform was also fabricated using delrin plastic and is equipped with lead wights. It also had a front and back part hinged at the ball of the foot. It is fully modifiable in that the height could be adjusted. The feel of the GEMS and the platform is similar with the exception of the generated backward motion.

\section{TESTING}

Subject testing consisted of three subjects who were all university student males, ages 20-25, with normal walking patterns in a study approved by the University of South Florida's Institutional Review Board. All three subjects 
were measured on their baseline walking pattern before walking on the GEMS. Temporal and spatial variables of gait were evaluated using the GAITRite Walkway System (CIR Systems, Inc., PA), which is a $2 \mathrm{ft}(0.6 \mathrm{~m})$ by $16 \mathrm{ft}$ $(4.9 \mathrm{~m})$ walkway consisting of pressure sensors that are able to accurately monitor each step position. Since we ultimately aim to even out asymmetric gait patterns, this study emphasizes the change in step length between baseline and immediately post-training, which is most relevant to the purpose of this study. The subjects were all healthy and start with only a small asymmetric gait.

For baseline measurement, each subject walked on the GAITRite Walkway System five separate times. The average step length of all five trials was taken and later compared to post-training step length. The baseline readings were analyzed for any initial asymmetry of the subject's gait before the GEMS was strapped to the foot with the shorter step length (if present). This was done because an individual with an asymmetric gait such as a stroke patient would have a shorter step length on the hemiplegic side, so in essence, the GEMS was attached to the "hemiplegic" leg of the healthy subject in order to increase the step length although the asymmetry was very small or nonexistent. In order to compensate for the height and weight of the GEMS, an adjustable platform was worn on the opposite foot.

The subject proceeded to walk back and forth on a $48 \mathrm{ft}$ $(14.6 \mathrm{~m})$ thin carpet walkway for approximately 20 minutes. The shoe was tested on a thin carpet surface in order to increase the friction between the smooth delrin plastic wheels and the ground, which prevented the shoe from slipping. During training, the subjects were observed and encouraged to take normal heel-to-toe steps in order to keep a consistent gait during the training process and between subjects.

After 20 minutes of gait training on the GEMS, the subject was seated in a rolling chair and the GEMS and support platform were removed. The subject was then rolled to the close-by GAITRite walkway system in order to capture the initial steps. The subject proceeded to walk five separate times on the walkway system where each trial was recorded for later comparison to the average baseline step length.

A retention test was also performed in order to observe if any after-effects persisted over a longer time period. This was done by letting the subject walk around at a comfortable pace without stopping for ten additional minutes. After the subject has walked ten minutes, the subject walked on the GAITrite mat five more times and an average retention step length was recorded for later analysis.

\section{Results And Discussion}

In pretesting, the Gait Enhancing Mobile Shoe (GEMS) pushed the wearer's foot back an average of 7 in $(17.8 \mathrm{~cm})$ in a continuous, steady, and non-jerky motion. The video associated with this paper shows the motion of the shoe. Due to its deformability, the shoe enables the wearer to toe off correctly for a smooth transition into the swing phase. Every step was very consistent and there was little variation, much like a split-belt treadmill. This low variation from step to step is important since it is our goal to mimic the motion of a split-belt treadmill

As mentioned before, the GEMS closely mimics a splitbelt treadmill, however, unlike the split-belt treadmill which has a tread speed ratio of $2: 1$, this version of the GEMS has a foot speed ratio of $4: 3$. Because of this difference we expect the after-effects to be smaller than the split-belt treadmill.

The difference in step length between the foot with the GEMS and the foot without are shown in Figure 6 for the baseline average, post training five trials of walking $16 \mathrm{ft}(4.9 \mathrm{~m})$, and retention average. Note that two out of the three subjects (subject 2 and 3) showed an increase in the asymmetry in the direction that we expected - the leg that wore the shoe developed a longer step length in the post-training trials. This implies that we are able to cause an adaptation in the gait patterns. The subject who had the opposite pattern also had the highest variability in step symmetry during baseline and retention testing, so there may be other effects affecting this subject's adaptation. The post-training average step length difference for subjects 2 and 3 increased 0.67 in $(1.72 \mathrm{~cm})$ and 0.94 in $(2.38 \mathrm{~cm})$, respectively. For both subjects 2 and 3 , the retention after a ten minute walking period was negligible, which is expected in healthy subjects.

Test subject 1 showed no average difference in step length increase, but rather a slight decrease of 0.36 in $(0.92 \mathrm{~cm})$ in the reverse direction. Looking at Figure 7, it becomes more apparent that the post training step length difference fluctuated around zero. The results for subject 1 become more interesting as they show that the average retention difference in step length has a magnitude of 1.10 in $(2.77 \mathrm{~cm})$ from baseline average in the reverse direction from the other two subjects. This deviation from our hypothesis can possibly be explained by the walking style of subject 1 . While subjects 2 and 3 comfortably walked in a correct gait when wearing the GEMS, subject 1 swung the leg with the GEMS around the side to compensate for the loss in step length, thus conditioning separate leg muscles. This indicates that training is dependent upon the wearer continuing to walk with a similar gait as they typically do.

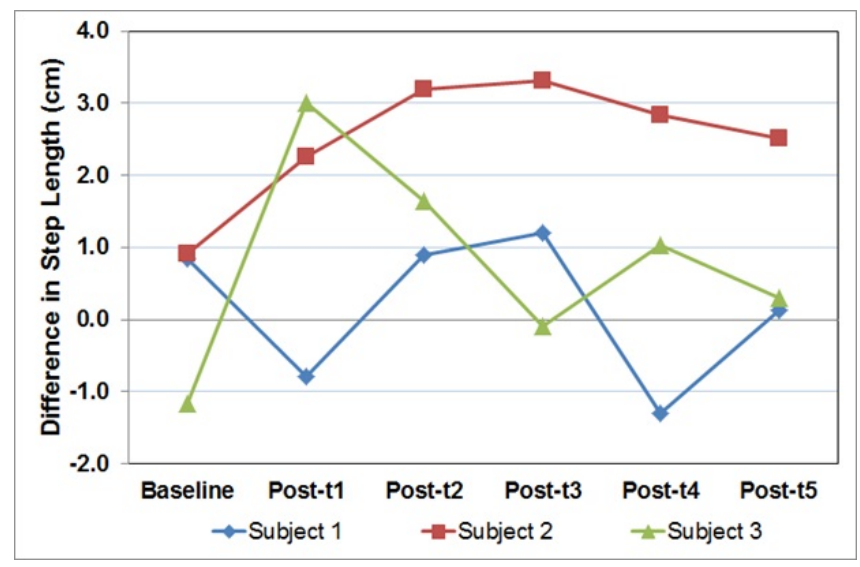

Fig. 6. Difference in step length for average baseline and the five walking trials in post-training. Notice that subject 1 exhibits variable aftereffects. 


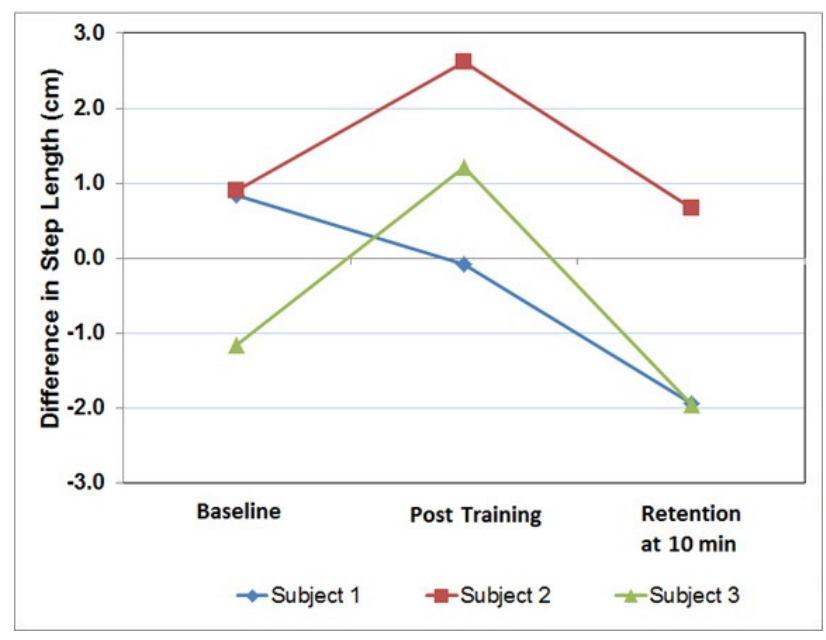

Fig. 7. Difference in step length for average baseline, average post-training, and retention after ten minutes of non-stop walking for all three subjects.

The baseline average and all five post-training trials are shown in Figure 7. Again, test subject 2 and 3 validate the hypothesis and show post training after-effects. Compared to their baseline difference in step length, these after-effects are very strong in subjects 2 and 3, although with slight differences. As subject 2 kept the after-effect over all five post-training trials, subject 3 diminishes some of the aftereffect at trial 3, regains some at trial 4, and then again diminishes at trial 5 .

These results are very promising in the area of asymmetric gait rehabilitation. However, further investigation is needed to completely validate the effectiveness of the GEMS. A larger study is needed to validate these results. The larger study will involve two shoes, one moving the foot in each direction, to double the applied asymmetry.

\section{CONCLusion AND Future Work}

We have successfully constructed a Gait Enhancing Mobile Shoe (GEMS), which is able to mimic a splitbelt treadmill in that it provided a smooth and consistent backward motion. Although the applied perturbation is smaller than that of a split-belt treadmill, our preliminary test results indicate that we are able to generate aftereffects in the same direction as those found in split-belt treadmill studies. The GEMS outlined in this paper builds upon the previous design concepts, which resulted in the current version that is lighter, smoother, and lower to the ground than previous versions.

The shoe generates the desired backward motion in a completely passive way with various benefits beyond the split-belt treadmill, such as enabling gait training in different environments and locations and long-term gait training over a longer period of time. These benefits will allow for further investigation of the long-term after-effects of longer training sessions, which we hypothesize will lead to longer retention of the corrected gait in individuals with asymmetric gaits.

Most importantly, the GEMS was found to demonstrate functional outcomes in its ability to alter normal gait patterns. In the study completed with this design, two out of three subjects validated our hypothesis and changed their post training difference in step length. This gives reason to further investigate the effects of the shoe. Future studies with the GEMS will include testing it on more subjects, letting test subjects walk further and longer in the shoe, using two shoes where one moves the foot forward and the other moves the foot backward, and further modifying and optimizing the shoe. In particular, the shape of the wheel can be optimized to vary the redirected force throughout the stance phase.

\section{ACKNOWLEDGEMENTS}

The authors thank Dr. Seok Hun Kim for his assistance. This work is funded by NIH grant \# 1R21HD066200-01.

\section{REFERENCES}

[1] D. Reisman, R. Wityk, and A. Bastian, Split-belt treadmill walking adaptation in post-stroke hemiparesis, J. Neurologic Physical Therapy, vol. 29, p. 196, 2005.

[2] T. Lam, M. Anderschitz, and V. Dietz, Contribution of feedback and feedforward strategies to locomotor adaptations, J. Neurophysiol, vol. 95, pp. 766-773, 2006.

[3] S. Morton and A. Bastian, Cerebellar Contributions to Locomotor Adaptations during Splitbelt Treadmill Walking, J. Neurosci., vol. 26, no. 36, pp. 9107-9116, 2006

[4] T. Lam, C. Wolstenholme, and J. F. Yang, How do infants adapt to loading of the limb during the swing phase of stepping? Journal of Neurophysiology, vol. 89, no. 4, pp. 1920-1928, 2003.

[5] D. Reisman, R. Wityk, K. Silver, and A. Bastian, Locomotor adaptation on a split-belt treadmill can improve walking symmetry post-stroke, Brain, vol. 130, no. 7, pp. 1861-1872, 2007.

[6] J. Krakauer, M. Ghilardi, and G. Ghez, Independent learning of internal models for kinematic and dynamic control of reaching. Nature Neuroscience, vol. 2, pp. 1026-1031, 1999.

[7] J.-S. Lou and J. R. Bloedel, A new conditioning paradigm: Conditioned limb movements in locomoting decerebrate ferrets, Neuroscience Letters, vol. 84, no. 2, pp. 185 - 190, 1988.

[8] D. S. Reisman, R. Wityk, K. Silver, and A. J. Bastian, Splitbelt treadmill adaptation transfers to overground walking in persons poststroke. Neurorehabil Neural Repair, vol. 23, no. 7, pp. 735-744, Sep 2009

[9] R. Keamey, Context dependence of intrinsic and reflex contributions to ankle stiffness, in Proc. of 1st International IEEE EMBS Conf. on Neural Engineering, March 2003, pp. 434-437.

[10] R. F. Reynolds and A. M. Bronstein, The broken escalator phenomenon aftereffect of walking onto a moving platform. Exp Brain Res, vol. 151, no. 3, pp. 301-308, Aug 2003.

[11] A. M. Bronstein, K. L. Bunday, and R. Reynolds, What the broken escalator phenomenon teaches us about balance. Ann N Y Acad Sci, vol. 1164, pp. 82-88, May 2009.

[12] A. de Groot, R. Decker, and K. B. Reed, Gait enhancing mobile shoe (GEMS) for rehabilitation, in Proc. Joint Eurohaptics Conf. and Symp. on Haptic Interfaces for Virtual Environment and Teleoperator Systems, March 2009, pp. 190-195.

[13] R. Grasso, L. Bianchi, and F. Lacquaniti, Motor Patterns for Human Gait: Backward Versus Forward Locomotion, J. Neurophysiol, vol. 80, no. 4, pp. 1868-1885, 1998.

[14] J. Perry, Gait Analysis: Normal and pathological function, N. S. Inc., Ed. Thorofare, 1992, vol. 50 .

[15] C. Honeycutt, J. Sushko, and K. B. Reed, Asymmetric passive dynamic walker, in Proc. IEEE Int. Conf. Rehabilitation Robotics, 2011, in press.

[16] D. Reinkensmeyer, J. Emken, and S. Cramer, Robotics, motor learning, and neurologic recovery, Annu. Rev. Biomed. Eng., vol. 6, pp. 497525, 2004.

[17] M. Whittle, Gait Analysis: An Introduction. Elsevier Health Sciences, 1998.

[18] K. L. Bunday and A. M. Bronstein., Visuo-vestibular Influences on the Moving Platform Locomotor Aftereffect", J Neurophysiol 99: 13541365,2008

[19] I. Handzic, E. Vasudevan, and K. B. Reed, "Motion Controlled Gait Enhancing Mobile Shoe for Rehabilitation," Proc. of the 12th Intl. Conf. on Rehabilitation Robotics (ICORR), Zurich, Switzerland, June, 2011. 